\title{
Compensações realizadas por crianças com paralisia cerebral espástica durante o levantar da cadeira
}

\author{
Compensations performed by children with spastic cerebral \\ palsy during the chair lift
}

\section{Compensaciones realizadas por niños con parálisis cerebral espástica durante la elevación de la silla}

\author{
Beatriz Calixto Silva ${ }^{1}$, Claudia R. M. Alcantara de Torre², Cristina dos \\ Santos Cardoso de Sá3
}

1.Graduanda em fisioterapia, Universidade Federal de São Paulo, campus Baixada Santista, Santos-SP, Brasil.

2.Fisioterapeuta, Mestre, especialista em fisioterapia neurofuncional, Instrutora do Conceito Neuroevolutivo, Santos-SP, Brasil.

3.Fisioterapeuta, Doutora, Professora do Departamento Ciências do Movimento Humano, UNIFESP, Campus Baixada Santista, Santos-SP, Brasil.

\begin{abstract}
Resumo
Objetivo. Identificar as compensações motoras realizadas por crianças com paralisia cerebral (PC) espástica diparéticas e hemiparéticas durante a tarefa de levantar da cadeira. Método. Estudo transversal descritivo. Avaliou as compensações motoras utilizando a parte inicial do teste Timed Up and Go (TUG). A avaliação foi filmada por duas câmeras (uma no plano sagital e outro no plano frontal) e posteriormente os vídeos foram analisados qualitativamente. Resultado. Amostra composta por oito crianças com PC espástica, cinco hemiparéticas $(\mathrm{GPCH})$ e três diparéticas (GPCD). Observou-se que todas as crianças realizaram algum tipo de compensação motora para realização da tarefa de forma que facilitasse o movimento e o objetivo final fosse atingido. Dentre essas compensações podemos destacar a utilização do apoio da cadeira ou dos membros inferiores (MMII) como impulso de movimento, abdução dos MMII, adução com rotação medial dos MMII, afastamento dos pés de forma a aumentar a base de suporte, aumento da flexão de joelho com a posteriorização dos pés e abdução de ombro com flexão de ombro ou de cotovelo. Conclusão. Não foi possível estabelecer os padrões das compensações que crianças com PC espástica hemiparéticas e diparéticas realizam na tarefa de levantar da cadeira de acordo com o grupo.
\end{abstract}

Unitermos. paralisia cerebral; criança; fisioterapia; postura sentada; posição ortostática

\footnotetext{
Abstract

Objective. The aim of this study was to identify the motor compensations performed by children with spastic diparetic and hemiparetic cerebral palsy (CP) during the task of stand up from a chair. Method. A descriptive cross-sectional study. Motor compensations were assessed with the initial part of the Timed Up and Go (TUG) test. The evaluation was filmed by two cameras, one in the sagittal plane and the other in the frontal plane, and later the videos were qualitatively analyzed. Results. In total, eight childrens with spastic cerebral palsy were evaluated, five hemiparetics (GPCH) and three diparetics (GPCD). All the subjects performed some type of motor compensation during the task, achieving the final goal. Among these compensations can be highlighted the movement impulse by using the chair support or the lower limbs, lower limbs abduction or adduction with medial rotation, the feet separation in order to increase the support base, increased knee flexion while the feet are set behind it and shoulder abduction with shoulder or elbow flexion. Conclusion. According to each group, it was not possible to establish the motor compensation standards performed by children with $\mathrm{CP}$ during the task of stand up from a chair.
} 
Keywords. Cerebral Pasly; child; Physical Therapy Specialty; sitting position; standing position

\begin{abstract}
Resumen
Objetivo. Identificar las compensaciones motoras que realizan los niños con parálisis cerebral diparética y hemiparética espástica (PC) durante la tarea de levantarse de la silla. Método. Estudio descriptivo transversal. Compensaciones de motor evaluadas usando la parte inicial de la prueba Timed Up and Go (TUG). La evaluación fue filmada por dos cámaras (una en el plano sagittal y la otra en el plano frontal) y posteriormente los videos fueron analizados cualitativamente. Resultados. Muestra compuesta por ocho niños con parálisis cerebral espástica, cinco hemiparéticos (GPCH) y tres diparéticos (GPCD). Se observó que todos los niños realizaban algún tipo de compensación motora para realizar la tarea de forma que facilitaba el movimiento y se alcanzaba el objetivo final. Entre estas compensaciones, podemos destacar el uso de sillón o de miembros inferiores como impulso de movimiento, abducción de miembros inferiores, aducción con rotación medial de MMII, separación de los pies para aumentar la base de apoyo, aumento flexión de rodilla con pie posterior y abducción de hombro con flexión de hombre o codo. Conclusión. No fue posible establecer los estándares de compensación que realizan los niños con parálisis cerebral espástica hemiparética y diparética en la tarea de levantarse de la silla según el grupo.
\end{abstract}

Palabras clave. Parálisis cerebral; niño; fisioterapia; sedestación; posición de pie

Trabalho realizado na Universidade Federal de São Paulo, campus Baixada Santista, Santos-SP, Brasil.

Endereço para correspondência: Cristina SC de Sá. Departamento de Ciências do Movimento Humano, Universidade Federal de São Paulo. Av. Ana Costa 95. Vila Mathias. Santos-SP, Brasil. CEP 11050-240. Email: cristina.sa@unifesp.br

\title{
INTRODUÇÃO
}

Paralisia cerebral (PC) é uma desordem neuromotora complexa resultante de lesão no cérebro em desenvolvimento que causa limitações nas atividades de vida diária1,2. Os distúrbios motores da PC são frequentemente acompanhados de distúrbios de sensação, percepção, cognição, comunicação e comportamento, por epilepsia e por problemas osteomusculares secundários ${ }^{1}$. A PC pode ser classificada em três grupos principais: espástica, atáxica e discinética, que são baseados em sinais neurológicos claros indicando patologia nos sistemas motores cerebrais ${ }^{3}$. 
A PC espástica é o tipo mais comum ${ }^{4}$. Ela pode ser subdividida de acordo com a topografia da lesão, sendo considerados hemiparéticos aqueles que possuem comprometimento em apenas um hemicorpo e diparéticos aqueles que apresentam maior comprometimento nos membros inferiores (MMII), podendo os membros superiores (MMSS) também estar acometidos. Devido às diferentes formas de classificação, Palisano et al. $(1997)^{5}$ desenvolveram o Sistema de Classificação da Função Motora Grossa (GMFCS) que propõe método preciso e confiável para classificar de forma padronizada o grau de comprometimento funcional dessa população. Essa classificação considera as habilidades motoras grossas da criança, capacidade de iniciar o movimento voluntário, mudança de posição, limitações funcionais e necessidade de mobilidade com dispositivos de assistência em relação à idade ${ }^{6}$.

Pode-se entender que para qualquer indivíduo realizar determinadas atividades é necessário desenvolver estratégias para manter o centro de gravidade dentro da base de suporte. Com o crescimento, a criança aprende selecionar as estratégias mais adequadas para determinadas tarefa7. A aprendizagem motora, então, é o resultado da interação entre $O$ indivíduo, a tarefa e o ambiente. A habilidade de recuperar a estabilidade após perturbações é finalizada, em crianças com desenvolvimento típico entre os 7-12 anos de idade, ou seja, é quando apresentam o mesmo padrão de um indivíduo adulto, porém nas crianças com PC essa habilidade pode estar atrasada ${ }^{8}$. Então, para realizar 
determinadas tarefas, crianças com PC realizam compensações motoras, visando uma maneira possível de executá-las.

Dessa forma, é importante entender a tarefa de levantar, que é uma atividade comum na vida diária que precede muitas outras, como por exemplo, caminhar e brincadeiras em pé. Esse movimento é a transição para a postura ereta que requer movimento do centro de massa de uma posição estável para uma menos estável sobre as extremidades inferiores estendidas, ou seja, de grande exigência biomecânica e com altos níveis de coordenação neuromuscular para regular essa transferência do centro de massa. Além disso, o SNC é desafiado a controlar a estabilidade e o alinhamento dos segmentos corporais ${ }^{9}$.

Diante do exposto, os objetivos desse estudo foram investigar as compensações motoras realizadas por crianças com PC espástica diparéticas e hemiparéticas e observar se há diferença entre os grupos durante a tarefa de levantar da cadeira.

\section{MÉTODO}

\section{Local do estudo}

Estudo transversal que foi realizado em centros especializados no tratamento de crianças com PC situados na cidade de Santos. Esta pesquisa foi submetida à Comissão de Ética e Pesquisa da Universidade Federal de São Paulo (UNIFESP) sob o parecer número 2.755.062. 


\section{Amostra}

Foram incluídas crianças de ambos os sexos, com idade entre 5 e 13 anos, sem déficit visual e/ou auditivo associado, e que compreenderam os comandos verbais simples necessários para a execução da tarefa, que cujos pais assinaram os termos de consentimento livre e esclarecido (TCLE), e o participante que teve condições também concordou em participar assinando o termo de assentimento (TA).

Não foram incluídas crianças com PC que possuíam deformidades que comprometem a permanência na postura ortostática, bem como aquelas que não atingiram essa postura, possuíam dificuldade para caminhar e levantar-se independentemente; crianças com PC relacionadas aos níveis III, IV e V do GMFCS; e crianças cujos pais não assinaram o TCLE, ou aquelas que não concordaram ou não quiseram participar do estudo apesar do consentimento dos pais.

\section{Procedimento}

As crianças foram convidadas a participar do estudo de modo que foram orientadas, previamente, sobre os procedimentos dos quais estariam sujeitas, bem como a importância deste estudo.

A avaliação envolveu o teste Timed Up and Go (TUG), instrumento que pode ser usado para avaliar equilíbrio, controle postural antecipatório, bem como a mobilidade funcional. Ele mede, em segundos, o tempo necessário para 
que o indivíduo se levante da cadeira com apoio de braços, caminhe $3 \mathrm{~m}$, vire-se, volte para a cadeira num percurso também de $3 \mathrm{~m}$, totalizando então os $6 \mathrm{~m}$, e sente-se novamente ${ }^{10}$.

O teste foi filmado por duas câmeras, uma posicionada no plano frontal, $2 \mathrm{~m}$ à frente do obstáculo onde a criança fez a volta para retornar à cadeira, e foi responsável por filmar uma parte da marcha no caminho de ida e volta, importante para a cronometragem do tempo de execução do teste; a outra foi posicionada à $90^{\circ}$ do plano sagital, também a $2 \mathrm{~m}$ de distância do indivíduo, e ficou responsável por filmar o momento em que a criança se levanta, importante para observar as compensações por ela estabelecidas.

Para realização do teste TUG a cadeira foi ajustada para cada criança para que mantivesse os pés apoiados no chão, garantindo que o tornozelo formasse um ângulo de $90^{\circ} \mathrm{com}$ a tíbia. Dessa forma, a criança se levantou, caminhou por $3 \mathrm{~m}$ em um percurso estabelecido por fita marcada no chão, delimitando largura de $1 \mathrm{~m}$, deu uma volta em objeto posicionado estrategicamente, e voltou $3 \mathrm{~m}$, em um trajeto também demarcado por fita, terminando o percurso sentando-se novamente na cadeira. $O$ trajeto foi demonstrado para cada criança antes da avaliação começar e após o esclarecimento de possíveis dúvidas. O avaliador cronometrou o teste desde o comando verbal de 'já' até a criança parar de se movimentar, sentando-se na cadeira. 
Cada criança foi instruída a caminhar em velocidade confortável e foi permitido que todos fizessem uma tentativa do teste para familiarizar-se com o procedimento.

Os vídeos foram analisados por um software de vídeo simples como o Windows Media Player, pelo qual foi possível fazer as análises qualitativas das compensações de movimento e, para isso, optou-se por utilizar apenas a parte inicial do teste TUG - o movimento de levantar-se da cadeira.

\section{Análise Estatística}

Foi realizada distribuição de frequência absoluta e percentílica para a variável compensação motora, ou seja, estratégia de movimento usada pelas crianças na realização da tarefa.

Foi realizada análise descritiva (média, desvio padrão e mediana) para o tempo de realização do TUG de acordo com o grupo (GPCH e GPCD), e para a descrição da amostra total.

Foi utilizado o software SPSS versão 23 para as análises descritivas.

\section{RESULTADOS}

O estudo foi composto por uma amostra de oito crianças com PC espástica; cinco hemiparéticas $(\mathrm{GPCH})$, duas à direita e três à esquerda; e três diparéticas (GPCD). Seis classificam-se como GMFCS I, e duas do GPCD classificamse como GMFCS II. A idade variou entre 5 e 13 anos e apenas uma é do sexo feminino, que compõe o GPCD. Duas crianças 
do grupo GPCD fazem o uso de órteses regularmente, mas a fim de padronizar a coleta pedimos para que realizassem a tarefa sem esses dispositivos assegurando que isso não Ihes trouxesse nenhum risco (Tabela 1 ).

Tabela 1. Caracterização da amostra por grupo.

\begin{tabular}{l|c|c|c|c|c}
\hline Grupo & Participante & Sexo & $\begin{array}{c}\text { Idade } \\
\text { (anos) }\end{array}$ & GMFCS & $\begin{array}{c}\text { Uso de } \\
\text { órtese }\end{array}$ \\
\hline \hline \multirow{3}{*}{ GPCH } & 1 & M & 8 & I & N \\
& 2 & M & 5 & I & N \\
& 3 & M & 9 & I & N \\
& 4 & M & 13 & I & N \\
& 5 & M & 13 & I & N \\
\hline \hline \multirow{3}{*}{ GPCD } & 6 & M & 12 & II & S \\
& 7 & F & 5 & I & N \\
& 8 & M & 5 & II & S \\
\hline
\end{tabular}

GPCH: Grupo de crianças com paralisia cerebral hemiparéticos; GPCD: Grupo de crianças com paralisia cerebral diparéticos; $\mathrm{F}=$ feminino; $\mathrm{M}=$ masculino; $\mathrm{S}=\operatorname{sim} ; \mathrm{N}=$ não.

Após a análise dos vídeos, pelo Software Windows Media Player, em que as crianças realizaram o levantar a partir da posição sentada (etapa inicial do teste TUG), observou-se as compensações de cada participante na execução da ação nos planos sagital e frontal. As compensações foram descritas em cada plano quando presente.

No plano sagital, durante o movimento de levantar-se da cadeira todas as crianças realizaram flexão de tronco, dando impulso ao movimento, o que é esperado para essa ação. Uma criança do GPCH apoiou seus braços nos MMII, 
dando impulso no momento de levantar-se; e duas do GPCD realizaram apoio dos MMSS no banco; uma criança apoiou o MSD e uma apoiou o MSE no banco, ambas do GPCH. O membro superior apoiado é o contrário à hemiparesia (Tabela 2).

Tabela 2. Distribuição de frequência absoluta e percentílica das compensações para realização do levantar da cadeira.

\begin{tabular}{|c|c|c|}
\hline Compensações & \multicolumn{2}{|c|}{ Grupos } \\
\hline Compensações no plano sagital & $\begin{array}{l}\text { GPCH } \\
\mathbf{N}(\%)\end{array}$ & $\begin{array}{l}\text { GPCD } \\
\mathbf{N}(\%)\end{array}$ \\
\hline Flexão de tronco para frente (impulso) & $5(100)$ & $3(100)$ \\
\hline Descarga de peso no pé contrário à hemiparesia & $1(20)$ & 0 \\
\hline Posterioriza um pé (contrário à hemiparesia) & $1(20)$ & 0 \\
\hline Apoio dos MMSS nos MMII & $1(20)$ & 0 \\
\hline Até $10^{\circ}$ de abdução de ombro com flexão de cotovelo bilateral & 0 & $1(33,33)$ \\
\hline Posterioriza os pés bilateralmente & $2(40)$ & $2(66,66)$ \\
\hline Apoio MMSS no banco & 0 & $2(66,66)$ \\
\hline $\begin{array}{l}\text { Até } 10^{\circ} \text { de abdução do ombro excessiva flexão de ombro } \\
\text { bilateralmente }\end{array}$ & 0 & $1(33,33)$ \\
\hline Compensações no plano frontal & $\begin{array}{l}\text { GPCH } \\
\mathbf{N}(\%)\end{array}$ & $\begin{array}{l}\text { GPCD } \\
\text { N(\%) }\end{array}$ \\
\hline Apoio MSD no banco & $1(20)$ & 0 \\
\hline Apoio MSE no banco & $1(20)$ & 0 \\
\hline Rotação medial MMII com adução do quadril & $3(60)$ & $2(66,66)$ \\
\hline Afastamento dos MMII & 0 & $1(33,33)$ \\
\hline Excessiva abdução MIE & $1(20)$ & 0 \\
\hline
\end{tabular}


Uma criança realizou descarga de peso no membro inferior contralateral à hemiparesia e apenas uma realiza posteriorização do pé (aumento de flexão do joelho), também contrário à hemiparesia. Isso possibilita que a descarga de peso seja feita no membro não acometido e facilite o movimento. Também houve esse aumento de flexão do joelho bilateralmente em duas crianças do GPCH e duas do GPCD (Tabela 2).

Em relação aos MMSS, podemos observar até $10^{\circ}$ de abdução do ombro com excessiva flexão do mesmo bilateralmente, em uma criança do GPCD e até $10^{\circ}$ de abdução de ombro com flexão de cotovelo bilateralmente em uma criança do mesmo grupo (Tabela 2).

No plano frontal, pode-se observar que três crianças do GPCH e duas do GPCD realizaram rotação medial dos quadris com importante adução do quadril, enquanto uma criança do GPCD realizou afastamento dos MMII, aumentando a base de suporte e uma criança do GPCH realizou abdução apenas do MIE, o membro acometido pela hemiparesia (Tabela 2).

O tempo do teste TUG foi medido por meio de um cronômetro simples, a fim de especulações com os dados normativos das crianças típicas para a mesma faixa etária (Tabela 3). Esses encontram-se acima dos valores médios apresentados por crianças típicas na mesa idade (os valores normativos para as idades 3-5 anos de crianças e adolescentes saudáveis são de 6,59 s, para as idades de 69 anos, de 5,69s e para as idades de 10 a 13 anos de 5,57s). 
Tabela 3. Tempo TUG para os grupos GPCH e GPCD.

\begin{tabular}{|c|c|c|c|}
\hline GRUPO & VOLUNTÁRIO & $\begin{array}{l}\text { TEMPO TUG } \\
\text { (seg,cent) }\end{array}$ & Análise descritiva \\
\hline \multirow{5}{*}{ GPCH } & 1 & 12,68 & \multirow{5}{*}{ 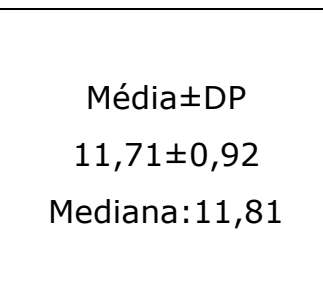 } \\
\hline & 2 & 12,81 & \\
\hline & 3 & 11,81 & \\
\hline & 4 & 11,26 & \\
\hline & 5 & 10,39 & \\
\hline \multirow{3}{*}{ GPCD } & 6 & 11,34 & \multirow{3}{*}{$\begin{array}{c}\text { Média } \pm \text { DP } \\
\text { 13,99 } \pm 1,52 \\
\text { Mediana:12,61 }\end{array}$} \\
\hline & 7 & 15,37 & \\
\hline & 8 & 12,61 & \\
\hline \multirow{2}{*}{\multicolumn{3}{|c|}{ TOTAL }} & Média \pm DP \\
\hline & & & Mediana: 12,61 \\
\hline
\end{tabular}

\section{DISCUSSÃO}

Esse estudo identificou as compensações realizadas por crianças com PC classificadas como hemiparéticas ou diparéticas na tarefa de levantar da cadeira. Porém, devemos salientar que não foi possível encontrar padrões de movimentos compensatórios entre os grupos estudados devido ao reduzido número de participantes.

$\mathrm{Na}$ análise dos vídeos, pudemos observar movimentos de tronco, MMSS e MMII que podem ser considerados compensatórios quando comparados aos movimentos típicos, esperados para esta tarefa e que de forma geral foram importantes para facilitar o movimento e concluir a tarefa da melhor forma possível. 
Crianças com PC apresentam diferenças significativas de comportamento motor, principalmente, quando comparadas entre os grupos, dependendo do comprometimento causado pela lesão. Apesar de essas diferenças serem possíveis de serem observadas na realização de tarefas motoras grossas, como por exemplo, na marcha, é preciso entender como de fato elas ocorrem entre os grupos. É importante destacar ainda que quanto melhor o controle postural no movimento de levantar-se, melhor é o desempenho durante a execução de atividades funcionais de mobilidade, e quanto melhor o controle postural da postura ortostática, melhor o equilíbrio ${ }^{11}$. Permitindo assim, perceber como é importante entender esses movimentos de forma clara e precisa, como foi realizado no presente estudo e que possui fácil aplicabilidade, já que pode ser realizado em qualquer ambiente clínico.

Durante a análise qualitativa dos vídeos pudemos observar que todas as crianças realizaram flexão de tronco para se levantar da cadeira, e apesar de ser um movimento esperado, não medimos a angulação em que foi realizada. Angulações excessivas de flexão de tronco na tarefa de levantar são uma tentativa de trazer seu centro de pressão mais próximo da base de suporte e então ganhar estabilidade, o que poderia ser uma consequência de comprometimento do controle postural ${ }^{12}$.

Associada à essa flexão de tronco, também pudemos observar o movimento dos MMSS em algumas crianças do 
GPCD, como abdução de ombro com flexão de cotovelo ou de ombro, que pode ser explicada a fim de facilitar 0 movimento, ajudando no impulso.

Durante a tarefa de levantar pudemos observar deslocamento do centro de pressão nos dois grupos de nosso estudo nas direções médio-lateral e látero-lateral, como também foi explorado com PC, que no geral, apresentaram maior deslocamento do centro de pressão na direção médiolateral em diferentes posições, quando comparadas às crianças típicas ${ }^{13}$. Observaram que esse deslocamento do centro de pressão está relacionado com o nível de funcionalidade dessas crianças, que demonstram deficiência para executar tarefas funcionais ${ }^{14}$. Mas é importante destacar que esse déficit pode estar relacionado com alteração de tônus ou de força que elas apresentam.

Ainda, sabe-se que essa compensação no deslocamento do centro de pressão está intimamente relacionada com as estratégias que essas crianças utilizam para a manutenção do equilíbrio, como por exemplo, a preferência do uso de musculatura do quadril. Crianças com PC que necessitam de apoio para levantarem-se da cadeira apresentaram menor oscilação/deslocamento do centro de pressão anteroposterior e médio-lateral do que outros voluntários que não utilizaram suporte, o que significa melhor controle postural durante o movimento, visto que o suporte provavelmente forneceu algum nível de estabilidade ${ }^{14}$.

Além disso, pudemos observar que uma criança do GPCH realiza posteriorização do pé (aumento da flexão de 
joelho), bem como faz descarga de peso no membro contrário à hemiparesia, facilitando o movimento de se levantar. Isso também foi observado ${ }^{11}$ e pode ser explicado pela provável existência de uma compensação intermembros durante a realização da atividade, em que o membro de apoio, responsável pela estabilidade e, portanto, o menos acometido compensa os déficits do membro contralateral.

A posteriorização dos pés bilateralmente, realizado por ambos os grupos, pode ser explicada, pois facilita gradualmente a execução da tarefa, melhorando o processo de aprendizado. Para crianças que não conseguem realizar essa tarefa, alguns comportamentos como esse aumento de flexão dos joelhos podem ser adotados nas terapias, para que, com a repetição, possam se tornar espontâneos e resultar em desempenho permanentemente aprimorado ${ }^{14}$. 0 aumento da base de suporte, com o afastamento dos pés também pode ser observado a fim de ajustar os MMII de forma que a tarefa proposta pudesse ser facilitada.

Em relação ao teste TUG, sabemos que não existe na literatura valores de referência para populações de crianças com PC na qual estamos estudando, mas os valores normativos para as idades 3-5 anos de crianças e adolescentes saudáveis são de 6,59 s, para as idades de 69 anos, de 5,69s e para as idades de 10 a 13 anos de 5,57 $\mathrm{s}^{15}$. Valores que podem ser comparados com os encontrados em nosso estudo apenas afim de especulações, visto que não foi realizada análise estatística para essa variável entre os 
grupos estudados, mas que podemos observar que a mediana dos valores de nossos participantes está acima dos dados normativos para crianças típicas na mesma faixa etária.

Além disso, o TUG demonstrou ser uma ferramenta capaz de avaliar a mobilidade funcional, o equilíbrio e a eficácia de reabilitação em crianças e adolescentes, além de poder ser correlacionado com outros testes específicos. Segundo a autora, o tempo de realização do teste pode ser explicado, ao menos em parte, em função da idade e do peso corporal $^{15}$.

Durante a tarefa proposta optamos por deixar os MMSS livres para que realizassem o movimento da forma como preferiram, a fim de que a tarefa fosse mais parecida com a que encontramos no dia a dia dessas crianças. Isso permitiu que algumas delas, de ambos os grupos, realizassem certo apoio desses membros, fossem eles no banco ou no colo (nos MMII), tanto uni quanto bilateralmente. Esse apoio serviu de suporte para que houvesse uma descarga de peso que facilitasse o movimento. Foi observado que quando as crianças possuíam suporte anterior para realização dessa tarefa, o déficit de força nos músculos extensores de joelhos e quadris foi compensado, houve redução da coordenação nos MMII, e dos déficits no controle postural dinâmico ${ }^{16}$. Com isso, podemos pensar que em nosso estudo esse apoio forneceu suporte mecânico para que as crianças realizassem a tarefa com melhor desempenho, principalmente se pensarmos nas crianças do GPCH, já que o membro apoiado 
era contrário à hemiparesia, ou seja, utilizando o suporte do membro não afetado para facilitar a tarefa.

É sabido que na PC espástica há comprometimentos musculoesqueléticos importantes, e dentre eles podemos destacar a adução e rotação interna dos quadris, excessiva flexão dos joelhos ${ }^{17,18}$. Isso pode explicar a rotação interna associada à adução do quadril encontrada nas crianças desse estudo, independente do grupo, quando realizam o movimento de se levantar. Também podemos entender que a fraqueza muscular geralmente encontrada nessa população explique a abdução do membro contrário à hemiparesia de uma criança do $\mathrm{GPCH}$, apesar de não termos medido a força muscular durante o estudo. Assim, há necessidade de analisar esses movimentos a fim de corrigir possíveis deformidades e ponderar um plano de tratamento utilizando fortalecimento da musculatura que se encontra enfraquecida e possibilitar que essas crianças tenham maior independência nas atividades de vida diária, conquistando assim, melhor qualidade de vida.

É sabido, de acordo com estudos anteriores, que as limitações físicas têm grande influência nas restrições de participação vivenciadas por crianças com PC e que melhor função física está correlacionada com melhor qualidade de vida $^{19}$. Por isso, conhecer suas estratégias e ajustes posturais é importante e auxilia a estabelecer um plano terapêutico mais individualizado para cada paciente, melhorando assim, sua funcionalidade. 
Nosso estudo se preocupou em entender quais alterações nas funções estão presentes nessas crianças, de forma que no processo de avaliação e reabilitação leve-se em consideração o quanto elas afetam suas vidas, atividades e participação. Em suma, podemos observar que as compensações motoras realizadas por crianças com PC hemiparéticas e diparéticas tiveram a finalidade de facilitar o movimento, permitindo que suas dificuldades fossem superadas e seu objetivo final - realizar a tarefa - fosse concluído com sucesso. Além disso, podemos observar que apesar dessas crianças, dentro dos grupos apresentarem a mesma topografia (hemiparéticas e diparéticas), podemos encontrar padrões de movimento muito diferentes entre elas, visto que existe grande variabilidade de características clínicas, mesmo quando classificadas pelo mesmo GMFCS.

Dessa forma, sugerimos que estudos como esses sejam feitos com mais participantes para que consigamos entender e identificar as estratégias de movimento utilizadas durante determinadas atividades, e possamos intervir a partir dessas avaliações, possibilitando traçar um plano terapêutico mais preciso e funcional para cada um.

O presente estudo traz como limitação o número reduzido de participantes, não permitindo que a análise pudesse ser feita de maneira mais abrangente. Sugerimos que para estudos posteriores, além de aumentar a amostra, seria pertinente a comparação entre crianças dos níveis GMFCS I e II, visto que apesar de possuírem independência 
funcional, apresentam comprometimentos motores substancialmente diferentes.

\section{CONCLUSÃO}

Podemos observar grande variabilidade entre as características clínicas das crianças com PC, o que dificulta padronizar um comportamento motor, apesar de serem classificadas nos mesmos níveis de GMFCS. Dessa forma, a partir dos achados encontrados, não foi possível estabelecer os padrões das compensações que crianças com PC espástica hemiparéticas e diparéticas realizam na tarefa de levantar da cadeira de acordo com o grupo.

\section{AGRADECIMENTOS}

Este projeto foi financiado pelo CNPq (Conselho Nacional de Desenvolvimento Científico e Tecnológico).

\section{REFERÊNCIAS}

1.Rosenbaum P, Paneth N, Leviton A, Goldstein M, Bax M, Damiano D, et al. A report: The definition and classification of cerebral palsy. Dev Med Child Neurol 2007;109:8-14. https://doi.org/10.1111/j.14698749.2007.tb12610.x

2.Gulati S, Sonchi V. Cerebral Palsy: An Overview. Indian J Pediatr 2017;85:1006-16. https://doi.org/10.1007/s12098-017-2475-1

3.Cans C, Dolk H, Platt MJ, Colver A, Prasauskiene A, Krägeloh-Mann $\mathrm{I}$, et al. Recommendations from the SCPE collaborative group for defining and classifying cerebral palsy. Dev Med Child Neurol Suppl 2007;109:35-8.https://doi.org/10.1111/j.1469-8749.2007.tb12626.x 4.Wimalasundera N, Stevenson VL. Cerebral Palsy. Pract Neurol 2016;16:184-94.https://doi.org/10.1136/practneurol-2015-001184 
5.Palisano R, Rosenbaum P, Walter S, Russell D, Wood E, Galuppi B. Development and reliability of a system to classify gross motor function in children with cerebral palsy. Dev Med Child Neurol 1997;39:214-23. https://doi.org/10.1111/j.1469-8749.1997.tb07414.x

6. Moreno-de-Luca A, Ledbetter DH, Martin CL. Genetic insights into the causes and classification of cerebral palsies. Lancet Neurol 2012;11:283-92. https://doi.org/10.1016/S1474-4422(11)70287-3

7.Vitor LGV, Silva Junior RA da, Ries LGK, Fujisawa DS. Controle postural em crianças com paralisia cerebral e desenvolvimento típico. Rev Neurocienc 2015;23:41-7.

https://doi.org/10.4181/RNC.2015.23.01.1016.7p

8.Allegretti KMG, Kanashiro MS, Monteiro VC, Borges HC, Fontes SV. Os efeitos do treino de equilíbrio em crianças com paralisia cerebral diparética espástica. Rev Neurocienc 2007;15:108-13. https://doi.org/10.34024/rnc.2007.v15.8701

9.Santos AN, Pavão SL, Rocha NA. Sit-to-stand movement in children with cerebral palsy: a critical review. Res Dev Disabil 2011;32:224352. https://doi.org/10.1016/j.ridd.2011.05.001

10. Nicolini-Panisson RD, Donadio MVF. Timed "Up \& Go" test in children and adolescents. Rev Paul Pediatr 2013;31:377-83. https://doi.org/10.1590/S0103-05822013000300016

11.Pavão SL. Controle postural e atividade sentado para de pé em crianças (Tese). São Carlos: Universidade Federal de São Carlos, 2012. https://repositorio.ufscar.br/handle/ufscar/5290

12.Pavão SL, Santos AN, Oliveira AB, Rocha NACF. Postural control during sit-to-stand movement and its relationship with upright position in children with hemiplegic spastic cerebral palsy and in typically developing children. Braz J Phys Ther 2015;19:18-25. http://dx.doi.org/10.1590/bjpt-rbf.2014.0069

13. Brianeze ACGS, Cunha AB, Peviani SM, Miranda VCR, Tognetti VBL, Rocha NACF, et al. Efeito de um programa de fisioterapia funcional em crianças com paralisia cerebral associado a orientações aos cuidadores: estudo preliminar. Fisioter Pesq 2009;16:40-5. https://doi.org/10.1590/S1809-29502009000100008

14. Medeiros DL, Conceição JS, Graciosa MD, Koch DB, Santos MJ, Ries LG. The influence of seat heights and foot placement positions on postural control in children with cerebral palsy during a sit-to-stand task. Res Dev Disabil 2015;43-44:1-10.

http://dx.doi.org/10.1016/j.ridd.2015.05.004

15. Nicolini-Panisson RDA. Valores normativos para o teste Timed "Up \& Go" em pediatria e validação para pacientes com Síndrome de Down (Tese). Porto Alegrre: Pontifícia Universidade Católica do Rio Grande do Sul - PUCRS, 2012. http://hdl.handle.net/10923/4643

16.Pavão SL, Arnoni JLB, Rocha NACF. Effects of Visual Manipulation in Sit-to-Stand Movement in Children With Cerebral Palsy. J Mot Behav 2018;50:486-91. https://doi.org/10.1080/00222895.2017.1367641

17.Cargnin APM, Mazzitelli C. Proposta de Tratamento Fisioterapêutico para Crianças Portadoras de Paralisia Cerebral Espástica, com Ênfase nas Alterações Musculoesqueléticas. Rev Neurocienc 2003;11:34-9. 
https://doi.org/10.34024/rnc.2003.v11.8892

18. Roque AH, Kanashiro MG, Kazon Sa, Grecco LAC, Salgado AS, OC $\mathrm{S}$. Análise do equilíbrio estático em crianças com paralisia cerebral do tipo diparesia espástica com e sem o uso de órteses. Fisioter Mov 2012;25:311-6. https://doi.org/10.1590/S0103-51502012000200008 19.Kerr C, McDowell B, McDonough S. The relationship between gross motor function and participation restriction in children with cerebral palsy: an exploratory analysis. Child Care Health Dev 2007;33:22-7. https://doi.org/10.1111/j.1365-2214.2006.00634.x 